\title{
35 challenges in materials science being tackled by Pls under 35(ish) in 2021
}

\section{Brian Aguado, ${ }^{1}$ Laura J. Bray, ${ }^{2}$ Sabina Caneva, ${ }^{3}$ Juan-Pablo Correa-Baena, ${ }^{4}$ Giuliana Di Martino, ${ }^{5}$ Chengcheng Fang, ${ }^{6}$ Yin Fang, ${ }^{7}$ Pascal Gehring, ${ }^{8}$ Gabriele Grosso, Xiaodan Gu, ${ }^{10}$ Peijun Guo, ${ }^{11}$ Yu He, ${ }^{12}$ Thomas J. Kempa, ${ }^{13}$ Matthew Kutys, ${ }^{14}$ Jinxing Li, ${ }^{15}$ Tian Li, ${ }^{16}$ Bolin Liao, ${ }^{17}$ Fang Liu, ${ }^{18}$ Francisco Molina-Lopez, ${ }^{19}$ Andrea Pickel, ${ }^{20}$ Ana M. Porras, ${ }^{21}$ Ritu Raman, ${ }^{22}$ Ellen M. Sletten,,23 Quinton Smith, ${ }^{24}$ Chaoliang Tan, ${ }^{25}$ Haotian Wang, ${ }^{26}$ Huiliang Wang, ${ }^{27}$ Sihong Wang, ${ }^{28}$ Zhongrui Wang, ${ }^{29}$ Geoffrey Wehmeyer, ${ }^{30}$ Lu Wei, ${ }^{31}$ Yuan Yang, ${ }^{32}$ Lauren D. Zarzar, ${ }^{33}$ Meiting Zhao, ${ }^{34}$ Yuqing Zheng, ${ }^{35}$ and Steve Cranford ${ }^{36, *}$}

Here we highlight 35 researchers approximately under the age of 35. Age, of course, is just a number-our target was emerging early-career academics. Contributors were recruited in a self-propagating "pay-it-forward" manner, with each invitee being suggested by a peer who had already contributed. The final collection is an inspiring look at the challenges the current generation of materials researchers are tackling.

One of the lesser-discussed impediments in academic publishing is the so-called "prestige bias"_our sights are typically set on those who have already been established and have a certain level of recognition and success. Many times, the reputations are welldeserved and based on significant development of and contribution to a research problem in the past. Careers and reputations are built upon achievements and accomplishments. In effect, prestige is gained retrospectivelyrecognizing one's impact and influence after the fact. With that in mind, Einstein was once attributed with saying:

"To raise new questions, new possibilities, to regard old problems from a new angle, requires creative imagination and marks real advance in science."
These new questions and possibilities arise from the newest generation of PIs and researchers-those who have yet to achieve the accomplishments and recognition of their mentors because they are currently laying the foundations for their own future accolades. Fresh takes, innovative ideas, and boundless energy are driving the next wave of innovations in materials science and across other disciplines.

Frequently, these ongoing trials and tribulations are overlooked and hard to uncover. How can we discover the next big thing when it hasn't happened yet? One does not simply invite a plenary speaker to discuss their future work.

To highlight just a snippet of these creative imaginations and endeavors, I decided to gather 35 challenges in ma-
1Department of Bioengineering, University of California, San Diego, San Diego, CA, USA

2School of Mechanical, Medical and Process Engineering, Queensland University of Technology (QUT), Brisbane, QLD, Australia

${ }^{3}$ Department of Precision and Microsystems Engineering, Delft University of Technology, Delft, the Netherlands

${ }^{4}$ Energy Materials Laboratories, School of Materials Science and Engineering, Georgia Institute of Technology, Atlanta, GA, USA

${ }^{5}$ Department of Materials Science and Metallurgy, University of Cambridge, Cambridge, UK

${ }^{6}$ Department of Chemical Engineering and Materials Science, Michigan State University, East Lansing, MI, USA

${ }^{7}$ School of Chemical and Biomedical Engineering, Nanyang Technological University, Singapore, Singapore

8 Institute of Condensed Matter and Nanosciences (IMCN), Université Catholique de Louvain, Ottignies-Louvain-la-Neuve, Belgium

${ }^{9}$ Advanced Science Research Center and The Graduate Center, City University of New York, New York, NY, USA

${ }^{10 S c h o o l ~ o f ~ P o l y m e r ~ S c i e n c e ~ a n d ~ E n g i n e e r i n g, ~}$ The University of Southern Mississippi, Hattiesburg, MS, USA

${ }^{11}$ Department of Chemical and Environmental Engineering \& Energy Sciences Institute, Yale University, New Haven, CT, USA

12Department of Applied Physics, Yale University, New Haven, CT, USA

13Department of Chemistry, Johns Hopkins University, Baltimore, MD, USA

${ }^{14}$ Department of Cell and Tissue Biology, University of California, San Francisco, San Francisco, CA, USA

${ }^{15}$ Department of Biomedical Engineering, Institute for Quantitative Health Science and Engineering $(\mathrm{IQ})$, Michigan State University, East Lansing, MI, USA

${ }^{16}$ School of Mechanical Engineering, Purdue University, West Lafayette, IN, USA

${ }^{17}$ Department of Mechanical Engineering, University of California, Santa Barbara, Santa Barbara, CA, USA

${ }^{18}$ Department of Chemistry, Emory University, Atlanta, GA, USA

${ }^{19}$ Department of Materials Engineering, $\mathrm{KU}$ Leuven, Leuven, Belgium

${ }^{20}$ Department of Mechanical Engineering, University of Rochester, Rochester, NY, USA

${ }^{21}$ Department of Biomedical Engineering, University of Florida, Gainesville, FL, USA

22Department of Mechanical Engineering, Massachusetts Institute of Technology, Cambridge, MA, USA

${ }^{23}$ Department of Chemistry and Biochemistry, University of California, Los Angeles, Los Angeles, CA, USA 
terials science being tackled by 35 researchers under the age of approximately 35. Actual age didn't really matter-I was simply looking for earlycareer tenure-track assistant-professor-level academics (or close to it-l know one contributor has recently been promoted to Associate). It hit me that most of my author interactions (particularly in the COVID era, where meeting new faces at conferences is limited) have been focused on more established Pls. Neither by design nor intent, I encountered my own form of prestige bias. Sure, I had a few close contacts and relationships with younger Pls, but not enough for 35 invitations. So I decided to let the next generation self-generate their own network of contributors-who else better to suggest emerging researchers than their own peers?

The idea was simple: I sent a mere three invitations to contribute a brief description of their own research and a recommendation of the next researcher to invite (see Figure 1). Three initial invitations were sent to three global regions in three distinct fields to attempt to limit any overlap as well as reflect a more diverse community (and the added benefit that the process would be slightly quicker with three concurrent invitation chains). My one request was that the recommended invite tackled a slightly different problem than one's own. This "pay-it-forward" approach both reduced any implicit bias I may have as an editor and also introduced me to many new researchers I have never interacted with. The end result was inspiring: 35 individuals across 32 institutions and 7 countries. Of note, the three repeated institutes (Michigan State, Rice, and Yale) resulted in suggestions from separate invitation chains (i.e., no one recommended a peer from the same institute).

After some bookkeeping, collating, and formatting, the resulting 35 challenges (in alphabetical order) are:
The Aguado iBiomaterials Lab uses biomaterial-based technologies to investigate biological mechanisms governing sex differences in cardiovascular disease. Sex differences in cardiovascular disease exist from the molecular to organism scales yet are rarely considered in the design of bioengineered disease models. My lab incorporates sex-specific features in our biomaterials-based in vitro and in vivo disease models to more closely recapitulate sex dimorphisms observed in cardiovascular diseases, specifically aortic valve stenosis. Our mission is to understand sex-specific mechanisms of cardiovascular disease and determine equitable treatment options for both women and men.

Brian Aguado, University of California, San Diego

My research program uses bioengineering to understand the heterogeneity of endothelial cells and the biological mechanisms that regulate blood vessel formation and function in different organs. Using biomimetic semi-synthetic hydrogels, the challenge is to engineer human 3D models that can mimic (to an extent) the complexity of the tissue microenvironment in situ and be applied to investigate blood vessels in normal and diseased states.

\section{Laura J. Bray, Queensland University of Technology}

Protein nanopores are the gatekeepers of the cell. They are narrow, fixed channels through which ions and small molecules are selectively transported. Macromolecules, however, are too large to shuttle into cells through these nanochannels. In our group, we are developing the first mechanically adaptable nanopores by uniquely combining DNA origami nanotechnology, machine-inspired design, and synthetic biology. By integrating structural adaptability, we aim to enable single- molecule studies of native (i.e., folded) proteins and protein aggregates, which will further both basic nanopore research and applied research in the field of molecular diagnostics.

\section{Sabina Caneva, TU Delft}

My group is using atomic/molecular layer deposition and thermal evaporation to develop a novel approach to grow atomic- and nano-scale metal halide perovskites with different dimensionalities. Crucial to this effort is my group's work to design new chemical reactions and processes involving small molecules that allow for the formation of hybrid organic/inorganic materials, which allow for better control of the materials' dimensionality because organic cations dictate the level of dimensionality. This work will lead to better design of the microstructure, which will ultimately help understand charge carrier

${ }^{24}$ Department of Chemical and Biomolecular Engineering, University of California, Irvine, Irvine, CA, USA

${ }^{25}$ Department of Electrical Engineering, City University of Hong Kong, Hong Kong

${ }^{26}$ Department of Chemical and Biomolecular Engineering, Rice University, Houston, TX, USA

${ }^{27}$ Department of Biomedical Engineering, University of Texas at Austin, Austin, TX, USA

${ }^{28}$ Pritzker School of Molecular Engineering, The University of Chicago, Chicago, IL, USA

${ }^{29}$ Department of Electrical and Electronic Engineering, University of Hong Kong, Hong Kong

${ }^{30}$ Department of Mechanical Engineering, Rice University, Houston, TX, USA

${ }^{31}$ Department of Chemistry, California Institute of Technology, Pasadena, CA, USA

${ }^{32}$ Department of Applied Physics and Applied Mathematics, Columbia University, New York, NY, USA

${ }^{33}$ Department of Chemistry, Penn State University, University Park, PA, USA

${ }^{34}$ Department of Chemistry, Institute of Molecular Aggregation Science, Tianjin University, Tianjin, China

${ }^{35}$ Institute of Microelectronics, Peking University, Beijing, China

${ }^{36}$ Editor-in-Chief, Matter, Cell Press, Cambridge, MA, USA

*Correspondence: scranford@cell.com

https://doi.org/10.1016/j.matt.2021.11.003 


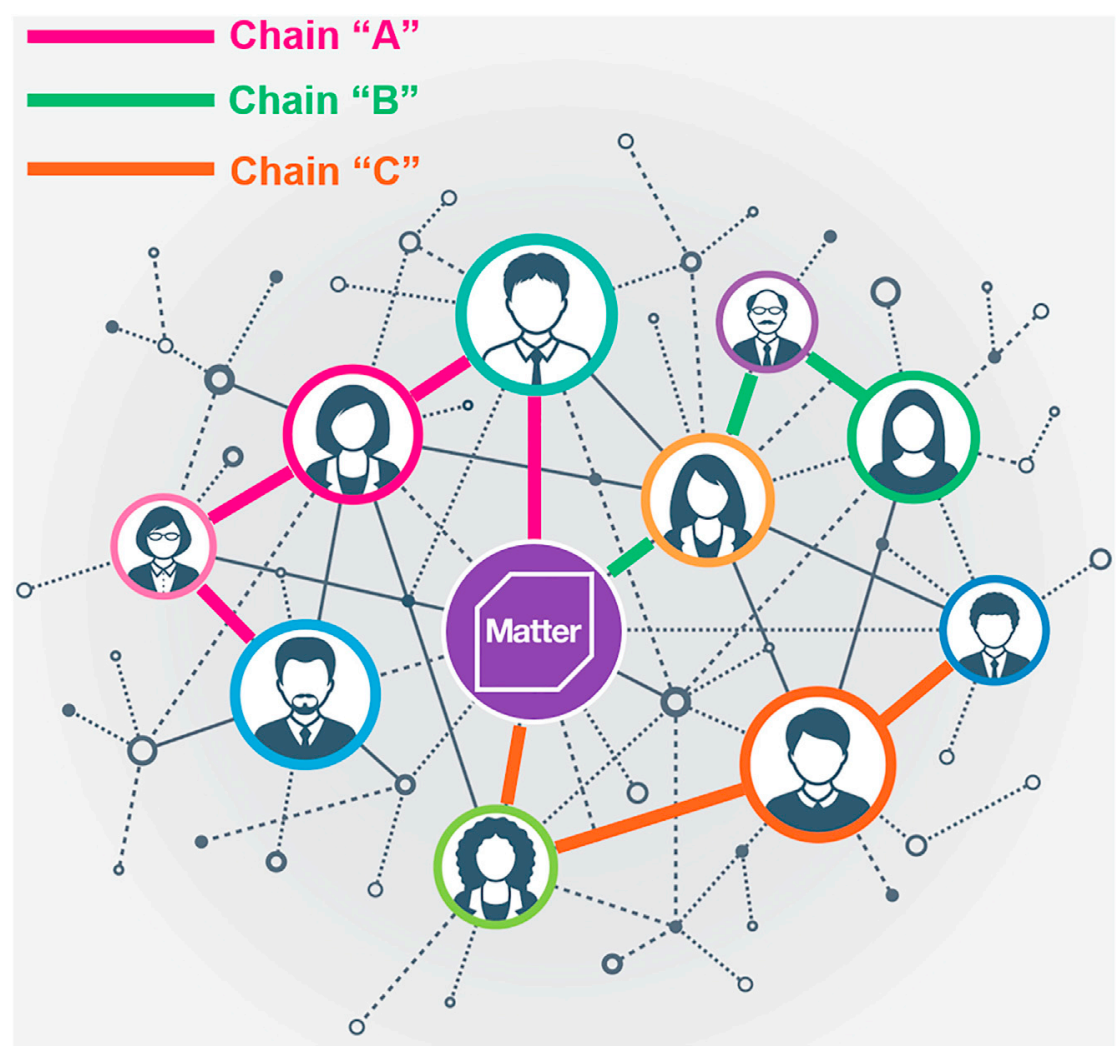

Figure 1. Pay-it-forward invitation chains

To recruit 35 contributions for this article, Matter only invited three individuals, who each suggested the subsequent invitee, thus extending the chain.

transport and recombination in the bulk and at interfaces in these materials.

Juan-Pablo Correa-Baena, Georgia Institute of Technology

My research (https://www. dimartinolab. msm.cam.ac.uk) links the fields of low-energy nanoscale device engineering and plasmon-enhanced light-matter interactions by implementing optically accessible devices. My research group uses the ultra-concentration of light to develop innovative fast ways to study real-time movement of individual atoms that underpins this new generation of ultra-low-energy memory nano-devices, thus overcoming the limitations of traditional investigation techniques and opening up new routes to sustainable future IT.

Giuliana Di Martino, University of Cambridge
Lithium metal batteries are the most promising next-generation energy storage technology that double the energy density of today's lithium-ion batteries. The dendrite growth and low cycling efficiency associated with lithium metal anodes are the most challenging issues to be tackled to enable this technology. Dr. Chengcheng Fang's research reveals the true failure mechanism of lithium metal anodes using multiscale quantitative characterization tools and attempts to achieve fully dense and reversible lithium metal anodes by using stack pressure control. These fundamental scientific discoveries pave the way for practical high-energy lithium metal batteries.

\section{Chengcheng Fang, Michigan State University}

Nowadays, materials scientists are solving the challenge of seamlessly inte- grating artificial materials and devices with biological tissues. In my opinion, tissue-like material building blocks and devices would be an ideal solution for solving the interface mismatch problem. The tissue-like systems would mimic the morphology, structure, and function of naturally occurring biological components using purely synthetic components or cellular-synthetic hybrid components. The tissue-like systems will play important roles in many current or emerging areas, such as bioelectronics, regenerative medicine, human-machine interfaces, and robotics. Current tissue-like systems usually display single or few biomimetic functions, such as self-healing and strain stiffening. In the future, materials scientists would create more sophisticated and highly integrated tissue-like functions-we hope to achieve this milestone soon!

Yin Fang, Nanyang Technological University

We investigate experimentally the fundamental physics of how heat and charge flow through nano-scale objects and how heat is converted into electricity in such materials. Our model systems are single-molecule devices or other low-dimensional materials, which offer the possibility to tailor their electronic structure by chemical design. By controlling physical properties, e.g., their spin-ground state, their entropy, or their quantum mechanical transmission probability, we aim at revealing mechanisms that make transport or energy conversion more efficient. This could ultimately allow us to develop new types of thermoelectric devices capable of harvesting electrical energy back from waste heat or precise local thermometers and spot-coolers for thermal management applications.

\section{Pascal Gehring, UC Louvain}

My research addresses the increasing demand for faster and more efficient ways to process and transfer 
information by using light. The goal is to provide a new level of scientific understanding of photonics quantum materials as well as the tools to produce them. My team is establishing a research activity to characterize and control with unprecedented precision solid-state emitters, such as excitons and atom-like systems, in atomically patterned two-dimensional materials. This research combines high-resolution transmission electron microscopy, advanced image analysis based on neural networks, high resolution imaging, and spectroscopy to create regular networks of active defects and phase domains in two-dimensional materials to study light-matter interactions in completely new systems.

\section{Gabriele Grosso, City University of New York}

As our societal need for better, greener, and more sophisticated materials grows, there must be advances in materials characterization tools and techniques to facilitate the new material development. There are challenges and opportunities associated with developing autonomous in situ characterization tools that can push the boundary for the time, spatial, and energy resolution as well as enable realtime characterization to study functional devices in operando and better interface with advanced theory and modern artificial intelligence algorithms to speed up new materials discovery. Our team studies the structure, dynamics, and morphology of functional polymers and aims to link their molecular structures to their macroscopic properties through advanced metrology with an emphasis on scattering techniques.

Xiaodan Gu, University of Southern Mississippi

We are developing new optical spectroscopic techniques, some of which would allow us to directly excite the vi- brations of materials and image the flow of energy in time. From these measurements we aim to gain fundamental understanding into energy and heat transport at the nanoscale for next-generation energy conversion and optoelectronic devices.

\section{Peijun Guo, Yale University}

Finding superconductors and magnets that operate at higher temperature and lower dimension with wider tunability is a perpetual pursuit in quantum materials. Combining high-resolution photoemission spectroscopy and $X$-ray scattering techniques, we scrutinize materials' electronic, magnetic, and lattice response under mechanical, electrical, optical, and chemical stimuli-directly in the materials' momentum space. Specifically, fluctuations of superconductivity, magnetism, or lattice structure are exploited for better control over material properties. The ultimate goal is to achieve property-specific materials engineering, guided by testable understanding of the microscopic mechanisms.

\section{Yu He, Yale University}

Precision synthesis of quantum materials is essential if they are to usher in a much anticipated revolution in information technology, metrology, and energy sustainability. My group develops innovative chemical methods to prepare two-dimensional inorganic crystals as building blocks of quantum materials. We elicit intriguing optical, catalytic, and electronic properties from these materials through atomically precise control of their dimensionality, structure, and strain state. Our ultimate goal is to assemble these precise building blocks into entirely new quantum systems that could have far-reaching impacts in optics, sensing, information processing, and energy conversion.

Thomas J. Kempa, Johns Hopkins University
My laboratory engineers new organotypic models of human tissues that offer experimental windows into typically inaccessible morphogenic events, such as developmental organogenesis and early events in cancer. By integrating designer biomaterials within biomimetic microfluidic systems, we are focused on achieving a molecular and physical understanding of how cellular perceptions of their surrounding microenvironment control tissuelevel behaviors by orchestrating cell behavior and fate decisions. An understanding of these mechanisms and their dysregulation offers the potential for new biomarkers and therapies for cardiovascular disease and cancer.

Matthew Kutys, University of California, San Francisco

Neurotransmitters play essential roles in regulating neural circuit dynamics both in the central nervous system as well as at the peripheral, including the gastrointestinal tract. However, bioelectronic neurotransmitter sensing tools that are capable of probing soft, complex, and actively moving organs are underdeveloped. I invented a tissue-mimicking stretchable neurochemical biointerface termed NeuroString, which is prepared by laser-patterning of a metal-complexed polyimide into an interconnected graphene/nanoparticle network embedded in an elastomer. NeuroString sensors allow chronic in vivo real-time multichannel and multiplexed monoamine sensing in the brain of behaving mouse, as well as measuring serotonin dynamics in the gut without undesired stimulations and perturbing peristaltic movements.

\section{Jinxing Li, Michigan State University}

The use of sustainable materials can be an effective strategy for the achievement of low or even negative emissions. Cellulose, the most abundant biopolymer on earth, features a 
hierarchically aligned structure from ångström- to macro-scale, which points to utilizing this biomaterial as low-dimensional building blocks with high performance and in advanced applications. We aim to unravel the multiscale process-structure-property relationships of cellulose to determine the fundamental mechanisms required to unlock the capabilities of this naturally nanostructured biomaterial.

\section{Tian Li, Purdue University}

Understanding the microscopic picture of energy transport and conversion in materials is essential for the development of next-generation electronics, renewable energy generation, and energy storage systems. My group strives to develop tools that can probe energy transport in materials at the smallest time and length scales. In particular, we are developing a scanning ultrafast electron microscope combining nanometer spatial resolution of electron microscopes and femtosecond time resolution of ultrafast lasers. With this powerful technique, we are now imaging and diagnosing the inner workings of emerging photovoltaic materials, hoping to provide design guidelines for more efficient solar cells.

Bolin Liao, University of California, Santa Barbara

My group is tackling the challenges in rapidly predicting molecular properties in real environments at the quantum mechanical level of theory to enable computational design and discovery under conditions of real energy science applications. Quantum mechanical (e.g., density functional theory) calculations usually do not account for the influence of environments, such as solvent and pressure, due to computation bottleneck. My group is developing fast and accurate quantum mechanical methods and machine learning models to describe molecules and materials in the solution phase or under extremely high pressure on the order of GPa.

\section{Fang Liu, Emory University}

Thermoelectric (TE) materials can convert waste heat into electricity and enable on-demand heating/cooling. To improve the applicability of TEs, we must go beyond improving their efficiency, since reducing fabrication cost and facilitating their implementation in different scenarios are equally important. My research addresses those challenges by targeting the development of printed and flexible TEs. Using printing technologies introduces a tremendous simplification in device fabrication. On the other hand, imparting mechanical flexibility to a device allows intimate contact with curved and large-area surfaces, maximizing the application of TEs to ubiquitous scenarios such as wrapping around pipes or attached to human skin.

\section{Francisco Molina-Lopez, KU Leuven}

Poor heat dissipation frequently limits the performance and reliability of modern electronic, data storage, and energy conversion devices. Identifying the root causes of thermal failure requires non-invasive temperature mapping techniques with nanoscale spatial resolution. We are addressing this challenge by harnessing new luminescent probes to develop far-field optical nanothermometry methods based on super-resolution spectroscopy, adapting powerful concepts that have transformed biological imaging for applications in the thermal sciences.

\section{Andrea Pickel, University of Rochester}

Until relatively recently, the role of the microbiome in modulating human health and disease was underappreciated; as a result, much remains to be elucidated regarding the mechanisms through which microbes regulate host health. In the Tissue-Microbe Interaction Lab, we are engineering in vitro disease models to explore the interactions between members of the microbiome and the natural biomaterials found in human extracellular matrix. We are particularly interested in studying the microbiomes of populations in the Global South. A detailed understanding of human-microbe interactions in these populations is crucial for the development of microbiome-based solutions to global health issues.

\section{Ana M. Porras, University of Florida}

Biological materials have an unparalleled ability to sense, process, and respond to their environment in real time, motivating their use as functional components in human-made systems. The Raman Lab creates adaptive biological materials powered by assemblies of living cells for applications ranging from medicine to machines. Currently, we are focused on engineering biological actuators to understand and replicate how biological creatures navigate dynamic unpredictable environments. We use biological materials and engineering tools to build living neuromuscular tissues that combine the compliant actuation of skeletal muscle with neural control and sensory feedback. These actuators help us understand and manipulate the architecture and function of the biological motor control system. Our goal is to restore mobility to those who have lost it after disease or trauma and to deploy biological actuators as functional components in machines.

Ritu Raman, Massachusetts Institute of Technology

Nanomaterials have long been hailed as the solution to biomedical challenges with opportunities for selective delivery, combination therapies, personalized medicine, and in situ synthesis. Despite all this potential, enormous optimization is still required for 
each material. We streamline the use of nanomaterials in diverse applications through the optimization of a nanoparticle platform that relies on the bioorthogonal fluorous phase. The self-segregating properties of perfluorocarbons provide a predictable and generalizable approach to load payloads into nanomaterials. We believe this focus on unique chemistries will enable convergent preparations and systematic studies of nanomedicines.

Ellen M. Sletten, University of California, Los Angeles

Traditional in vitro approaches that rely on static, planar surfaces fail to recapitulate the three-dimensional developmental niche that controls embryonic development, tissue maturation, and homeostasis. My research aims to phenocopy the mechanical and architectural complexity found in embryogenesis by leveraging soft biomaterials and microfluidics (enabled through engineering approaches). With this approach, human-induced pluripotent stem cells can be coaxed to differentiate to functional cellular constructs that can augment or replace disease tissues in a clinical setting.

Quinton Smith, University of California, Irvine

Layered two-dimensional (2D) materials have been recently proven to be promising absorbers for fabrication of high-performance infrared photodetectors. The key advantage of layered 2D materials is the absence of surface dangling bonds since layers stack together through van der Waals interaction. However, the lack of 2D semiconductors with suitable bandgaps and atomically thin body induced ultra-low absorption in 2D layers are the two major challenges for fabrication of high-performance 2D material-based infrared photodetectors. The research in my group aims to tackle the challenge by combining design/synthesis of novel narrow-bandgap alloyed 2D semiconductors with nanooptics design/ integration.

Chaoliang Tan, City University of Hong Kong

My group's research is mainly focused on designing advanced catalytic materials and novel electrolyzers to efficiently convert atmospheric molecules, such as $\mathrm{CO} 2, \mathrm{O} 2, \mathrm{H} 2 \mathrm{O}$, and $\mathrm{N} 2$, into valuable chemicals and fuels using renewable electricity as a green and sustainable chemical production route. Over the past few years our group has successfully developed a series of high-performance and low-cost catalysts including $\mathrm{Ni}$ single atom catalysts, $\mathrm{Bi}$ nanosheet catalyst, and $\mathrm{Cu}$ nanocube catalyst to selectively reduce $\mathrm{CO} 2$ into different products. We also developed a unique solid electrolyte reactor that enables a direct and continuous generation of pure liquid fuels from $\mathrm{CO} 2$ reduction without the need of downstream product purification process.

\section{Haotian Wang, Rice University}

Recent developments in optogenetics have demonstrated that modulation of the targeted neurons or specific projections in the brain is capable of rescuing behavioral deficits associated with Parkinson's disease, autism, epilepsy, and Alzheimer's Disease. However, optogenetics faces two major challenges for their clinical applications. First, due to limited tissue penetration of visible light, invasive craniotomy and intracranial implantation of optical fibers are generally required. Second, this approach requires genetic modification of neurons via viral transduction, which poses significant safety challenges. My group is developing approaches to solve these challenges.

Huiliang Wang, University of Texas at Austin
In the rapidly emerging field of human-integrated electronics, including wearable and implantable electronics, the main challenge is the mismatch of physicochemical properties between functional materials/devices and biological systems. As a consequence, the current wearable devices cannot provide effective and/or accurate monitoring to all the physiological signs (e.g., blood pressure and biomarker information), and the implantable electronics have limited spatial resolution while causing significant health risks. To solve this suite of challenges, innovative designs of electronic materials need to incorporate the fundamental understanding of material-biology interactions and possess biomimetic and biocompatible mechanical, topological, and chemical properties together with advanced electronic functions.

Sihong Wang, The University of Chicago

The advancement of $\mathrm{Al}$ relies on the hardware. However, traditional siliconbased computers are facing a performance bottleneck. Their physically separated memory and processing units incur large energy and time overheads in running Al. This is further intensified by the slowdown of Moore's law that has fueled the past development of electronics. Thus, a fundamental change to the computing paradigm is needed. Resistive switching materials can encode information in highly scalable devices thanks to the underlying chemical and physical reactions. In addition, they can compute with simple physical rules right at where the data is stored, mitigating the need to shuttle data, which may pave the way for future Al hardware.

\section{Zhongrui Wang, The University of Hong Kong}

Regulating the temperature of batteries, electronics, and buildings is currently energy-intensive and relatively inefficient. Many thermal 
researchers are working to develop thermally functional devices with a switchable thermal resistance; these devices would be reversibly cycled between a thermally conducting and thermally insulating state and could be triggered by a temperature change or by an applied field. New advances in solid-state, rapid, compact, and inexpensive heat switching would allow thermal engineers to simultaneous reduce the power consumed for heating in a cold environment while also improving reliability by preventing overheating in a hot environment.

\section{Geoffrey Wehmeyer, Rice University}

In our lab, one of the challenges we aim to tackle is to develop new spectral-imaging strategies to perform in operando high-resolution characterizations of the materials properties, which would be essential to inform the design of the next-generation materials. We utilize stimulated Raman scattering spectro-microscopy for highly non-perturbative and fast imaging of the fundamental processes to offer unknown dynamics information at the microscopic level, including the formation dynamics and structural-functional properties of bio-engineered materials and diffusion-reaction processes during electrochemical $\mathrm{CO}_{2}$ conversion and capture.

Lu Wei, California Institute of Technology

My research is focused on designing radiative cooling materials to reduce electricity usage and $\mathrm{CO} 2$ emission in thermal management. Radiative cooling materials have unique broadband optical properties to maximize reflection in the solar spectrum and thermal emission in the infrared transparent window of the atmosphere, so they lose heat to outer space and cool themselves without electricity, even under strong sunlight. I am combining simulations and experiments to understand structure-property correlation in radiative cooling materials, achieve directional and spectral selectivity to maximize performance, and produce radiative cooling materials in a scalable and low-cost way.

\section{Yuan Yang, Columbia University}

We are trying to understand how to design materials that exhibit "life-like" characteristics, such as the ability to move, adapt, and interact. This quest goes beyond the design of responsive materials, which change properties by transitioning between two different equilibrium states in response to an external trigger. Living materials instead persist in non-equilibrium states for long times and can continuously respond to fluctuations in their environment. While life itself is extraordinarily complex, we aim to uncover simpler approaches for combining chemistry and structure in ways that facilitate functional control over the non-equilibrium dynamic behaviors of materials.

\section{Lauren Zarzar, Penn State University}

For porous materials, such as metalorganic frameworks (MOFs) and covalent-organic frameworks (COFs), in many cases, owing to the lack of longrange ordering caused by small crystal size and poor crystallinity, it is not suitable for structure analysis using traditional techniques (e.g., single-crystal X-ray diffraction). How to determine the real crystal structure of low-dimensional MOF and COF nanomaterials is challenging, which is also the footstone for constructing reliable structure-performance relationships for their applications in research areas including adsorption, separation, catalysis, energy, etc.

\section{Meiting Zhao, Tianjin University}

Flexible electronics that seamlessly interact with biological systems pave the way toward precise personal healthcare monitoring and therapeutics. The flourishing development of flexible electronics is relied on the innovation of multi-functional materials and devices. We introduce abundant intermolecular interactions to organic functional materials, endowing them with controlled characteristics, such as stimulus responsiveness, bio-specificity, and biodegradability, for their application in next-generation electronics.

\section{Yuqing Zheng, Peking University}

Thirty-five early career researchers. Thirty-five challenges and problems being tackled in materials science. Dozens of graduate students to teach and postdocs to train. Hundreds of future manuscripts and articles to publish. The challenges represent future accomplishments and invitations to the NAS, Royal Society, or CAS (or geographical equivalent). Sometimes it is more inspiring to look at the challenges ahead than the solutions of the past. Remember, all current accomplishments were once just problems to solve.

What's yours? 